\title{
A Spin-down Power Threshold for Pulsar Wind Nebula Generation?
}

\author{
E. V. Gotthelf
}

Columbia Astrophysics Laboratory, Columbia University, 505 West 120th Street, New York, NY 10027, USA

\begin{abstract}
A systematic X-ray survey of the most energetic rotationpowered pulsars known, based on spin-down energy loss rate, shows that all energetic pulsars with $\dot{E}>\dot{E}_{c} \approx 4 \times 10^{36} \mathrm{ergs} \mathrm{s}^{-1}$ are X-ray-bright, manifest a distinct pulsar wind nebula (PWN), and are associated with a supernova event, with over half residing in shell-like supernova remnants. Below $\dot{E}_{c}$, the $2-10 \mathrm{keV}$ flux ratio $F_{P W N} / F_{P S R}$ decreases by an orderof-magnitude. This threshold is consistent with the lower limit on the spectral slope $\Gamma_{\min } \approx 0.6$ observed for rotation-powered pulsars (Gotthelf 2003). The apparent lack of bright PWNe below $\dot{E}_{c}$ suggests a change in the particle injection spectrum and serves as a constraint on emission models for rotation-powered pulsars. Neither a young age nor a high density environment is found to be a sufficient condition for generating a PWN, as often suggested, instead $\dot{E}$ is likely the key parameter in determining the evolution of a rotation-powered pulsar.
\end{abstract}

\section{A Chandra Study of the Most Energetic Pulsars}

Table 1 presents the 28 most energetic pulsars from the ATNF pulsar catalog, ${ }^{1}$ ordered by spin-down power $\dot{E}(=I \omega \dot{\omega}$, where $I$ is the neutron star moment of inertia and $\omega$ is its angular velocity). These include all known pulsars detected in both radio and X-ray with $\dot{E}>1.8 \times 10^{36} \mathrm{ergs} \mathrm{s}^{-1}$ (except one millisecond pulsar in this range). Twenty-five objects are radio pulsars and 21 are X-ray pulsars, of which only three are detected in X-rays alone. So far, five radio pulsars have no known follow-up yet in any waveband. For each pulsar with available Chandra ACIS X-ray data, and for its PWN, we measured the unabsorbed flux in the 2$10 \mathrm{keV}$ band using the method described in Gotthelf (2003). Here we compared these fluxes with $\dot{E}$ and present the flux ratio $F_{P W N} / F_{P S R}$, where $F_{P S R}$ is the sum of the pulsed and unpulsed pulsar emission.

All of the top 13 pulsars in Table 1 have been observed in X-rays, including the nine brightest X-ray PWNe used in the initial study of Gotthelf (2003). When ordered by $\dot{E}$ it is apparent that all pulsars with $\dot{E} \gtrsim 4 \times 10^{36} \mathrm{ergs} \mathrm{s}^{-1}$ are X-ray-bright, show a resolved PWN, and are associated with evidence of a supernova event. The jury is still out on PSR J1617-5055, which is highly

\footnotetext{
${ }^{1}$ See http://www.atnf.csiro.au/research/pulsar/catalogue .
} 
absorbed and was observed with Chandra too far off-axis to resolve a nebula, and on J1112-6102, for which no follow-up X-ray observation currently exists.

Table 1: Pulsars ordered by spin-down power.

\begin{tabular}{|c|c|c|c|c|c|c|}
\hline Pulsar & Remnant & $\begin{array}{c}\dot{E} \\
\times 10^{36} \\
\left(\operatorname{ergs~s}^{-1}\right)\end{array}$ & $\begin{array}{l}\text { Dist }^{a} \\
(\mathrm{kpc})\end{array}$ & $\begin{array}{c}\epsilon^{b}= \\
L_{X} / \dot{E}\end{array}$ & $\begin{array}{c}F_{P W N} / \\
F_{P S R}\end{array}$ & Code $^{c}$ \\
\hline J0537-6910 & N157B & 481.6 & 49 & 0.003 & 15 & $s-x$ \\
\hline $\mathrm{J} 0534+2200$ & Crab (SN1054) & 440.6 & 2.0 & 0.03 & 30 & $\operatorname{srx}$ \\
\hline J0540-6919 & SNR $0540-69$ & 146.5 & 49 & 0.05 & 4 & $\operatorname{srx}$ \\
\hline $\mathrm{J} 0205+6449$ & $3 \mathrm{C} 58(\mathrm{SN} 1181)$ & 27.0 & 3.2 & 0.0004 & 60 & srx \\
\hline $\mathrm{J} 2229+6114$ & $\mathrm{G} 106.3+2.7$ & 22.5 & 12 & 0.001 & 9 & $-r x$ \\
\hline J1513-5908 & MSH $15-52$ & 17.7 & 5.0 & 0.01 & 5 & $\operatorname{srx}$ \\
\hline $\mathrm{J} 1617-5055$ & & 16.2 & 6.5 & 0.001 & $\cdots$ & $-r x$ \\
\hline J1124-5916 & G292.0+1.8 & 11.9 & 5.4 & 0.0002 & 10 & $-r x$ \\
\hline $\mathrm{J} 1930+1852$ & $\mathrm{G} 54.1+0.3$ & 11.6 & 5 & 0.002 & 5 & $\operatorname{srx}$ \\
\hline J1420-6048 & Kookaburra & 10.4 & 7.7 & 0.004 & 10 & $-r x$ \\
\hline J1846-0258 & Kes 75 & 8.3 & 19 & 0.15 & 23 & $s-x$ \\
\hline J0835-4510 & Vela SNR & 6.9 & 0.3 & 0.0001 & 9 & $\operatorname{srx}$ \\
\hline J1811-1925 & G11.2-0.3 & 6.4 & 5 & 0.006 & 9 & $s-x$ \\
\hline $\mathrm{J} 1112-6103$ & & 4.5 & $\ldots$ & .. & . & $-r-$ \\
\hline $\mathrm{J} 1952+3252$ & CTB 80 & 3.7 & 2.5 & 0.0005 & 1.1 & $-r x$ \\
\hline J1709-4429 & G343.1-2.3? & 3.4 & 2.5 & 0.0001 & 3.5 & $-r x$ \\
\hline $\mathrm{J} 2021+3651$ & & 3.4 & 10 & $\ldots$ & $\ldots$ & $-r ?$ \\
\hline $\mathrm{J} 1524-5625$ & & 3.2 & 3.8 & & $\ldots$ & $-r ?$ \\
\hline $\mathrm{J} 1913+1011$ & & 2.9 & 4.5 & & . & $-r ?$ \\
\hline J1826-1334 & & 2.9 & 4.1 & 0.0008 & 2.3 & $-r x$ \\
\hline J1801-2451 & & 2.6 & 4.6 & 0.0008 & 0.1 & $-r x$ \\
\hline J1016-5857 & & 2.6 & 9.3 & $\ldots$ & $\ldots$ & $-r x$ \\
\hline $\mathrm{J} 1105-6107$ & & 2.5 & 7.1 & & & $-r-$ \\
\hline $\mathrm{J} 1119-6127$ & G292.2-0.5 (radio) & 2.3 & 4 & 0.00005 & 0.2 & $-r x$ \\
\hline $\mathrm{J} 1803-2137$ & & 2.2 & 4.0 & $\ldots$ & .. & $-r x$ \\
\hline J1048-5832 & & 2.0 & 3.0 & $\ldots$ & $\ldots$ & $-r x$ \\
\hline J1837-0604 & & 2.0 & 6.2 & 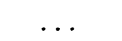 & $\ldots$ & $-r ?$ \\
\hline J0940-5428 & & 1.9 & 4.3 & . & $\ldots$ & $-r ?$ \\
\hline
\end{tabular}

${ }^{a}$ Best estimate of the pulsar distance from the literature.

${ }^{b}$ Efficiency, the ratio of pulsar luminosity $\left(L_{X} \equiv F_{X} 4 \pi d^{2}=L_{P W N}+L_{P S R}\right)$ in the $2-10 \mathrm{keV}$ band, following the procedure of Gotthelf (2003), and $\dot{E}$.

${ }^{c} \mathrm{~s}=$ Chandra survey PWN object (Gotthelf 2003); $\mathrm{r}=$ radio source; $\mathrm{x}=\mathrm{X}$-ray source.

In contrast, pulsars with $\dot{E}<\dot{E}_{c} \approx 4 \times 10^{36} \mathrm{ergs} \mathrm{s}^{-1}$ lack both a bright nebula and a supernova association in the X-ray energy regime. For several of these objects, Chandra observations detect weak nebulosity. Diffuse X-ray emission is found around PSRs J1709-4420 (Gotthelf, Halpern \& Dodson 2002) and $\mathrm{J} 2021+3651$, a newly discovered pulsar with a similar $\dot{E}$ (Roberts, these proceedings). An extremely faint X-ray "tail" is found trailing the "Duck" radio pulsar PSR J1801-2451, but this is interpreted as a ram-pressured confined cometary wind (Kaspi et al. 2001). The Chandra observation of PSR J1826-1334 confirms a faint PWN, barely resolved with the ROSAT HRI (Finley, Srinivasan \& Sangwook 1996). Finally, arcsecond localization of J1105-6107, previously 

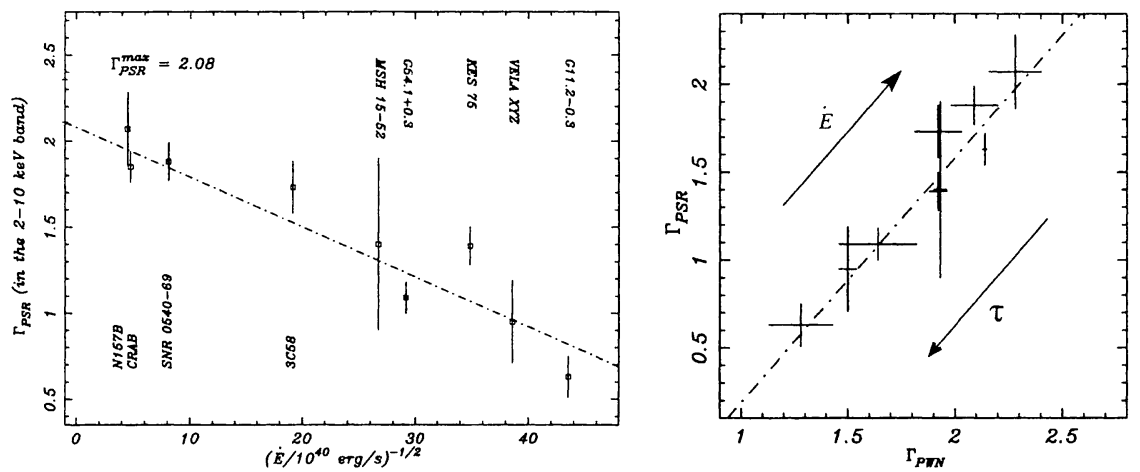

Figure 1. Left panel: A comparison between the 2-10 keV spectral slope of the nine brightest known pulsars $\left(\Gamma_{\mathrm{PSR}}\right)$ and $\dot{E}_{40}^{-1 / 2}$, with $\dot{E}$ in units of $10^{40} \mathrm{ergs} \mathrm{s}^{-1}$. The dashed line indicates the best-fit model. Right panel: Relationship between the above pulsars' $\Gamma_{\mathrm{PSR}}$ and that of their wind nebulae $\left(\Gamma_{\mathrm{PWN}}\right)$, assuming a simple power-law spectral model. The dashed line indicates the best-fit. The physical origin of this relationship has yet to be determined. From Gotthelf (2003).

associated with X-ray emission (Gotthelf \& Kaspi 1998), shows that the X-rays originate from an unrelated nearby source.

Evidently all pulsars with $\dot{E}>\dot{E}_{c}$ display bright PWNe while for the less energetic pulsars the nebular emission is vestigial, at best, when resolved from the background. This fact is quantified by the flux ratio $F_{P W N} / F_{P S R}$ given in Table 1 which shows that the PWNe of the less energetic pulsars are genuinely sub-luminous relative to their PSR flux. This comparison is best done statistically since the distance estimates are mostly uncertain (factor $\sim 2$ ). Above $\dot{E}_{c}$, the average flux ratio for these pulsars is of order $\sim 14$, while the less energetic pulsars have a ratio of order $\sim 1.5$. This factor of 10 change in the relative X-ray flux cannot be explained as a distance bias, as the range of distances overlap between the less and more energetic pulsars (see Table 1).

A possible explanation for a critical $\dot{E}_{c}$ is provided in Gotthelf (2003), where the spectra of the most energetic pulsars are shown to depend on $\dot{E}-$ the more energetic the pulsar, the steeper its spectrum. This relation is given by $\Gamma_{\mathrm{PSR}}=\Gamma_{\max }+\alpha \dot{E}^{-1 / 2}$ with a minimum observed $\Gamma_{\min } \approx 0.6$ (see Fig. 1). Most interestingly, $\Gamma_{\min }$ corresponds to $\dot{E}_{c} \approx 4 \times 10^{36} \mathrm{ergs} \mathrm{s}^{-1}$, right at the observed threshold for bright PWNe. Since the spectral index likely reflects the spectrum of the injected wind particles (Pacini \& Salvati 1973a, b), a critical phenomena in the acceleration process may be responsible for the observed threshold, perhaps turning off the pulsar wind or the PWN shock and allowing the nebula to fade with time and/or $\dot{E}$. For this fossil PWN, the above $\Gamma$ vs. $\dot{E}^{-1 / 2}$ relationship likely becomes invalid; some evidence for this is provided by preliminary spectra of faint PWNe belonging to the less energetic pulsars. 
The basic result presented here is also seen in the radio band where only the most energetic rotation-powered pulsars are found to display a radio PWN (Cohen et al 1983; Frail \& Scharringhausen 1997; Gaensler et al. 2000). The $\dot{E}_{c}$ threshold is also found to be applicable at these wavelengths, as none of the less energetic pulsars display a radio PWN at all, despite a sensitive search at 1.4 $\mathrm{GHz}$ around 27 pulsars with $1.2 \times 10^{32}<\dot{E}<2.8 \times 10^{36} \mathrm{ergs} \mathrm{s}^{-1}$ by Gaensler et al. (2000). Possible exceptions are PSRs J0908-4913, a pulsar with weak $\left(F_{P W N} / F_{P S R}<1 / 16\right.$ at $\left.1.2-2.2 \mathrm{GHz}\right)$, barely resolved radio emission (Gaensler et al. 1998), and J1856+0113 in SNR W44 with an apparent PWN. The latter object, however, is unusual and its exact nature requires further study.

Because $\dot{E}$ of the pulsars discussed here is unlikely to be correlated with local density, density is not a key factor for producing a detectable radio PWN as often claimed. Nor is a young age likely a sufficient condition for generating a PWN, considering the example of PSR J1119-6127, a 1600-yr-old pulsar in the radio shell G292.2-0.5 lacking a PWN (e.g. Crawford et al. 2001).

\section{Conclusions}

The main conclusions of this study are: (a) $\dot{E}$ is a key evolutionary parameter for rotation-powered pulsars; (b) a threshold exists, $\dot{E}_{c} \approx 4 \times 10^{36} \mathrm{ergs} \mathrm{s}^{-1}$, below which the generation of a PWN is greatly reduced (in X-rays) and/or undetected (in radio); (c) a Crab-like pulsar is defined as a rotation-powered pulsar with $\dot{E}>\dot{E}_{c} ;$ (d) a young age or a high local density environment is not a sufficient condition for generating a PWN, as often suggested.

Acknowledgments. This research is funded by LTSA NAG 5-7935.

\section{References}

Cohen, N. L., Cotton, W. D., Geldzahler, B. J., \& Marcaide, J. M. 1983, ApJ, 264,273

Crawford, C., Gaensler, B. M., Kaspi, V. M., Manchester, R. N., Camilo, F., Lyne, A. G., \& Pivovaroff, M. J. 2001, ApJ, 554, 152

Finley, J. P., Srinivasan, R., \& Park, S. 1996, ApJ, 466, 938

Frail, D. A., \& Scharringhausen, B. R. 1997, ApJ, 480, 364

Gaensler, B. M., Stappers, B. W., Frail, D. A., \& Johnston, S. 1998, ApJ, 499, L69

Gaensler, B. M., Stappers, B. W., Frail, D. A., Moffett, D. A., Johnston, S., \& Chatterjee, S. 2000, MNRAS, 318, 58

Gotthelf, E. V., \& Kaspi, V. M. 1998, ApJ, 497, L29

Gotthelf, E. V., Halpern, J. P., \& Dodson, R. 2002, ApJ, 567, L125

Gotthelf, E. V. 2003, ApJ, 591, 361

Kaspi, V. M., Gotthelf, E. V., Gaensler, B. M., \& Lyutikov, M. 2001, ApJ, 562, L163

Pacini, F., \& Salvati, M. 1973a, ApJ, 186, 249

- 1973b, Astrophys. Lett., 15, 39 